\title{
Papel del docente y de la escuela en el fortalecimiento de los proyectos de vida alternativos (PVA)
}

The Role of Teachers and the School in the Strengthening of Alternative Life Projects (ALP)

O Papel do professor e da escola no fortalecimento de projetos de vida alternativos (PVA)

\section{Karen García-Yepes* iD orcid.org/0000-0001-6433-2264}

Para citar este artículo: García-Yepes, N. (2020). Papel del docente y de la escuela en el fortalecimiento de los proyectos de vida alternativos (PVA). Revista Colombiana de Educación, 1(79). https://doi.org/10.17227/rce.num79-7453

$\begin{array}{rr}\text { (c) (7) } & \text { Recibido: } 22 / 01 / 2018 \\ \text { BY } & \text { Evaluado: } 04 / 03 / 2018\end{array}$

* Doctora en Ciencias Sociales y de la Educación, Universidad Autónoma Latinoamericana, Medellín, Colombia. 


\section{Resumen}

Este artículo surge de una tesis doctoral, cuyo objetivo es analizar el papel del docente y del sistema educativo en el fortalecimiento de los proyectos de vida alternativos (PVA) de los estudiantes. Como metodología se recurrió a la investigación de tipo cualitativo, y la técnica de recolección de información fue la entrevista en profundidad, donde participaron docentes y directivos de una institución educativa colombiana. Con respecto a los resultados, se evidencia que los procesos educativos en contextos vulnerables están condicionados por las limitaciones sociales y económicas del entorno, así como por las características del sistema educativo tradicional. En cuanto a las conclusiones, se plantea que el papel del docente y del sistema educativo consiste en aportar prácticas pedagógicas innovadoras como alternativa a la visión instrumental de la educación. Estas estrategias deben orientarse hacia el impacto en las mentalidades del estudiante y a la interacción entre el aprendizaje de conocimientos y habilidades para la vida, con el fin de incidir de manera significativa en la construcción de IOS PVA.

\section{Palabras clave}

contexto social; contexto educativo; problemas sociales; papel del docente; papel del estudiante

\section{Keywords}

social environment; educational environment; social problems; teacher role; student role

\begin{abstract}
This article is the result of a doctoral thesis that aimed to analyze the role of teachers and the educational system in the strengthening of the Alternative Life Projects (ALP) of the students. The methodology used was the qualitative research method, and in-depth interviews with teachers and directors of a Colombian school were used to gather information. Results show that educational processes in vulnerable contexts are conditioned by the social and economical limitations of the environment, as well as the characteristics of the traditional educational system. As for the conclusions, it is suggested that the role of teachers and the educational system is to provide innovative pedagogical practices as an alternative to the instrumental vision of education. These strategies must be oriented towards the impact on the mindset of the student and the interaction between the learning of knowledge and abilities for life with the purpose of significantly influencing the construction of ALP.
\end{abstract}

\section{Resumo}

Este artigo surge de uma tese de doutorado, cujo objetivo é analisar o papel do professor e do sistema educacional no fortalecimento dos projetos de vida alternativos (PVA) dos alunos. Como metodologia, utilizamos a pesquisa qualitativa e a entrevista em profundidade como técnica de coleta de informações, na qual participaram professores e diretivos de uma instituição de ensino colombiana. Com relação aos resultados, evidenciamos que os processos educacionais em contextos vulneráveis são condicionados pelas limitações sociais e econômicas do ambiente, bem como pelas características do sistema educacional tradicional. Em relação às conclusões, colocamos que o papel do professor e do sistema educacional é proporcionar práticas pedagógicas inovadoras como alternativa à visão instrumental da educação. Essas estratégias devem ser orientadas para o impacto nas mentalidades do aluno e a interação entre o aprendizado de conhecimentos e habilidades para a vida, a fim de influenciar significativamente a construção dos PVA.

\section{Palavras-chave}

contexto social; contexto educacional; problemas sociais; papel do docente; papel do estudante 


\section{Introducción}

El objetivo de este artículo es analizar el papel del docente y del sistema educativo en el fortalecimiento de los proyectos de vida alternativos (PVA) de los estudiantes. Este concepto surge como resultado de una tesis doctoral titulada "El papel de la Escuela en el fortalecimiento de los Proyectos de Vida Alternativos (PVA): su aporte en el proceso del posconflicto y al desarrollo comunitario en Colombia". De acuerdo con dicha investigación, el PVA hace referencia a un proceso alternativo donde la vida académica de la niñez y la adolescencia se alterna con las responsabilidades laborales de la vida adulta. En este sentido, la búsqueda de las metas personales y la colaboración con el sustento del hogar se complementa con el proceso de formación en el ámbito escolar.

Este análisis es importante por cuanto el papel de la escuela consiste en desarrollar habilidades para la vida y para relacionarse socialmente. En ese sentido, es pertinente replantear la mirada sobre su rol, ya que en los últimos años el debate sobre la función social de la educación fue retomado por parte de entidades estatales de orden nacional y departamental en Colombia. No obstante, en algunos casos esto se ha traducido en la implementación de modelos educativos descontextualizados que requieren mayor coherencia con las necesidades de las comunidades.

En estas circunstancias entran en juego dos modelos que son contradictorios entre sí. Por un lado, el basado en los planteamientos de las pedagogías críticas y transformadoras, que hacen énfasis en la necesidad de promover procesos educativos que favorezcan el desarrollo de las potencialidades del estudiante. Por otro, el que se asienta en las perspectivas de las pedagogías conservadoras, donde se favorece la acumulación de conocimientos. En ambos casos, hace falta hacer mayor énfasis en las necesidades particulares de los estudiantes de acuerdo con las características del contexto social y las limitaciones del sistema educativo concreto donde se desarrolla un proceso educativo. Por tanto, es necesario fomentar un modelo educativo alternativo y con sentido humanista, que promueva el desarrollo de habilidades y valores que complementen la dimensión intelectual, humana y social del individuo, a la vez que fortalezca su papel como ciudadano crítico y activo.

Así, cabe preguntarse el papel que cumplen el docente y la escuela, particularmente en contextos vulnerables. Eso es importante en la medida en que se requieren estrategias pedagógicas, didácticas y curriculares que respondan a las prácticas educativas tradicionales, donde el docente es una figura que representa la autoridad y genera aprendizajes que son unilaterales, verticales y acumulativos. Sin embargo, también se requiere que la implementación de propuestas críticas y activas en el aula sea 
contextualizada a las realidades sociales y educativas de estudiantes que, en algunos casos, aún requieren aprender conocimientos de acuerdo con su grado escolar. En esa medida, se podrá hacer énfasis en el desarrollo de habilidades por parte del estudiante como estrategia para favorecer su acceso a nuevas etapas de la educación obligatoria y permitir la aplicación de esos aprendizajes en contexto. Esto como alternativa para responder a un sistema educativo donde las prácticas tradicionales reproducen las desigualdades en cuanto a los procesos de aprendizaje entre el sistema público y el privado, creando diferentes oportunidades en el acceso a niveles formativos avanzados y al mercado de trabajo. Todo ello como apuesta para incidir de manera significativa en la construcción de los proyectos de vida alternativos (PVA).

\section{Fundamentación teórica}

\section{Las pedagogías transformadoras}

La investigación se fundamenta en los principios teóricos centrados en las pedagogías transformadoras. De acuerdo con sus objetivos, se realiza un análisis crítico de las estructuras sociales y sus problemáticas con el fin de proponer acciones educativas orientadas a la transformación económica, política, cultural y social (Ayuste, 2011; Ayuste y Trilla, 2005). En este sentido, la educación y la labor docente adquieren una función social orientada a la generación de mayores oportunidades sociales. Por ello, las pedagogías transformadoras convierten al educando en protagonista de su proceso de formación. Por su parte, el docente no es un transmisor de conocimientos, sino un líder que promueve la construcción del conocimiento en favor del empoderamiento de los estudiantes. Por el contrario las pedagogías conservadoras se caracterizan por validar los valores establecidos, las tradiciones culturales y las estructuras sociales (Ayuste, 2011).

Desde esta perspectiva, las pedagogías transformadoras abordan el centro educativo no como una entidad externa al contexto social, sino como un componente integral de la comunidad. En este caso, las personas no adquieren un único papel como estudiantes, pues la escuela deviene en espacio donde ellos actúan como ciudadanos, haciendo ejercicio activo de sus deberes y derechos. Así, estas pedagogías se enfocan en convertir al aula de clases en entorno de construcción colectiva del conocimiento, debate social, formación integral y desarrollo comunitario.

Entre estas pedagogías, a lo largo de varias décadas, se han fundamentado diferentes propuestas teóricas y metodológicas. En esta investigación, se toman como base aquellas en las que se promueve la crítica social y la construcción colectiva del conocimiento, como ejes de la transformación. 
En esa medida, es pertinente destacar la pedagogía crítica, la educación emancipatoria, el aprendizaje dialógico y el constructivismo crítico. Además, se destaca un especial interés en su relación con las pedagogías prácticas, cuyos planteamientos teóricos proponen un cambio efectivo de las realidades existentes (Ayuste, 2011).

\section{La pedagogía crítica y su papel en los procesos de aprendizaje crítico y activo}

La pedagogía crítica destaca la importancia de la labor docente en la generación de reflexiones sociales y políticas desde las aulas con el fin de motivar acciones de cambio en comportamientos y actitudes frente a las problemáticas de un contexto. Según su planteamiento, la escuela está inscrita en la comunidad y debe fomentar análisis que promuevan acciones de transformación frente a las problemáticas de una sociedad (Ayuste, Flecha, Palma y Lleras, 1994). De este modo, los salones se convierten en espacio de ejercicio de la ciudadanía donde se comienza a impulsar la participación activa y la acción propositiva frente a la desigualdad, la violencia o la exclusión. En esa medida, esta pedagogía no es neutral y apuesta por el cambio social a través del compromiso de personas críticas frente las realidades en las que se desenvuelven (Boyce, 1996; Freire, 1997; Giroux, 2014; McLaren, 2005).

Desde esta perspectiva, la pedagogía crítica se convierte en proceso que al cambiar críticamente al sujeto, también modifica sus relaciones con el mundo, al que se siente en deber y capacidad de transformar. En esa medida, esta propuesta promueve el aprendizaje, la reflexión y la acción en beneficio de las comunidades empobrecidas, marginadas y excluidas (McLaren, 2005). En esta pedagogía, sin embargo, el proceso del saber no se asume como conjunto de hechos transmitidos sino como construcción, creación y reconstrucción que permite una nueva comprensión de las problemáticas sociales. De esta perspectiva, se genera una transformación del estudiante, de su esencia y de su papel en la sociedad. Adquiere una renovada comprensión de su contexto y un nuevo compromiso social que lo impulsa a buscar activamente la equidad de oportunidades y la superación de las desigualdades. Así, la educación crítica impulsa el desarrollo de habilidades, valores y competencias que favorecen la comprensión del mundo y su intervención sobre él, en favor de la justicia social, política y económica (Freire, 1997; Giroux, 2014).

En este contexto, esta propuesta pedagógica evidencia el conocimiento como un proceso de construcción social, ciudadana y continua enfocada en la comprensión de sí mismo y del mundo. Por tanto, las relaciones sociales, las problemáticas y las desigualdades adquieren una nueva significación ante un saber que ha sido construido comunitariamente y en 
el marco del contexto al que pertenece. Pues en efecto, la pedagogía crítica se basa en la idea de que el proceso educativo debe fomentar la construcción colectiva del aprendizaje a partir de la interacción crítica, creadora y reflexiva de estudiantes y docentes (Giroux, 2014; Goodman, 2008; McLaren, 2005). En estas circunstancias, el conocimiento no es un saber único, unilateral y completo sobre nosotros mismos y sobre la sociedad. Por el contrario, es una creación que, en su calidad de acción, también es un acto de transformación. Según Freire, se trata de un proceso colectivo donde los que enseñan y los que aprenden, construyen conjuntamente una nueva significación frente al saber (1970, 1993, 1997). Por ello, el conocimiento no es transferencia, no es acumulación, no es depósito y tampoco es banco de saberes. A través de esta dinámica, el estudiante, en tanto que individuo se reconoce como parte activa en la creación de percepciones frente a un hecho determinado.

Desde este enfoque, en cuanto creación, el conocimiento es más que una construcción teórica e impulsa la acción. Es actuación reflexiva sobre la realidad que cambia los comportamientos frente a las desigualdades y las injusticias. Por eso, se responde a las necesidades del estudiante y se crean las condiciones para su autodeterminación en la sociedad, actuando proactivamente en favor de derechos que fortalecen la democracia (Giroux, 2014, McLaren, 2005). He ahí entonces una alternativa para comprenderse a sí mismo y el papel que cada uno cumple en la sociedad. En efecto, el individuo vuelve a recrearse a través de este proceso como sujeto socialmente activo que hace ejercicio de sus derechos y deberes ciudadanos. De esa forma, se fomenta el compromiso y la responsabilidad. Es decir, el estudiante no es solo un espectador de las desigualdades, sino que es capaz de comprender sus razones y actúa para empoderarse de su proyecto de vida. Además, no sólo es capaz de identificar las razones de un conflicto, sino que reflexiona sobre él y mejora su convivencia en el aula. Se trata de cambios progresivos que transforman una comunidad.

De acuerdo con los planteamientos de la pedagogía crítica, la construcción del aprendizaje se realiza en el marco de una relación dialógica donde las interacciones entre estudiantes y docentes están mediadas por el diálogo. Sin embargo, este se convierte en reflexión y acción colectiva frente a las problemáticas de la sociedad. Por esta razón, todo proceso educativo que se basa en la discusión como estrategia para construir el saber tiene entre sus objetivos la mediación activa en las problemáticas de la cotidianidad (Casamitjana, Puigvert, Soler y Tortajada, 2000; Grundy, 1987; Freire, 1993). Por ello, enseñar y aprender, como interacción que renueva a las personas, constituyen un acto creador y que transforma las realidades de personas que se comprometen a desarrollar nuevas relaciones basadas en la solidaridad, el respeto y la inclusión. Allí comienzan los cambios que evidencian la educación como proceso de transformación 
humana. En las aulas, en su función de microcosmos social que favorece la reflexión sobre sí mismo y sobre el otro, impulsando acciones en favor de la convivencia social. Según Freire (1976), todo proceso educativo implica el análisis, problematización, diálogo, toma de decisiones, adquisición de compromisos y certeza de que la realidad es cambiante y corresponde al ser humano empoderarse de ese proceso.

Con esta perspectiva, la labor educativa no solo busca garantizar una formación integral del ser humano, sino también desarrollar competencias, habilidades y valores que contribuyan con la justicia social. Así, este tipo de educación pretende lograr mayor equidad a través del empoderamiento de mujeres, niñas, poblaciones vulnerables, minorías étnicas, víctimas del conflicto y comunidades empobrecidas. Así, el proceso de formación pasa a convertirse en un modo para fortalecer la construcción del yo y del proyecto de vida en beneficio de la comunidad. Por tanto, la educación es emancipadora, liberadora y esperanzadora. Los seres humanos construyen entre sí una nueva comprensión de los conocimientos, de las problemáticas y de las desigualdades a través de la reflexión crítica. A través del análisis y de la acción, se reconstruyen, se liberan y transforman su contexto (Freire, 1970).

De ese modo, la educación fomenta la esperanza sobre la posibilidad de construir realidades donde las mujeres puedan obtener mejores salarios, las niñas puedan ir a los centros educativos, los jóvenes consigan empleos y las minorías étnicas no sean excluidas. En este caso, la tarea educativa, a partir de ese ideal, fomenta concreta y objetivamente las condiciones para que las comunidades comprendan estas desigualdades y se apropien de sus decisiones. En efecto, cuando el docente promueve prácticas pedagógicas participativas y críticas, el estudiante adquiere competencias para comprender las realidades, interactuar con los demás y actuar en favor de sus objetivos. Como ciudadano, ya no se va a sentir irremediablemente atrapado por las dificultades económicas, políticas y sociales, sino que va a resurgir con una nueva actitud emancipadora. Se sentirá capaz y competente para lograr sus metas personales y laborales. Sabrá que es actor social determinante en la búsqueda de la equidad, la justicia y la inclusión. Esa es la esencia de una educación esperanzadora que promueve el ejercicio de la ciudadanía y la construcción de la democracia a través de la crítica y de la acción en favor de la transformación social e individual (Freire, 1993; Giroux, 2014; McLaren, 2005).

Los planteamientos desarrollados sobre la pedagogía crítica han encontrado en la educación popular latinoamericana otra fuente de teorización. De acuerdo con esta propuesta, el conocimiento se construye colectivamente a partir del diálogo, de la práctica y de la participación, teniendo en cuenta el contexto como eje de acción y de transformación (Núñez, 2005). En este caso, el educando se convierte en eje del proceso

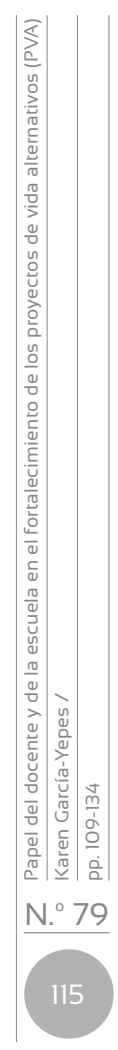


de formación y se intenta responder a sus necesidades e intereses con el fin de lograr un pleno desarrollo integral. Se aspira a que el estudiante se reconozca como sujeto activo del proceso educativo y se comprometa con el logro de sus propios objetivos. Desde este punto de vista, las relaciones de desigualdad se convierten en oportunidades que transforman al alumno y sus interacciones con el mundo. Pues en efecto, a través del conocimiento como acción dialéctica, él se empodera y transforma su calidad de vida. Por ese motivo, la educación se manifiesta como acto democratizador que emancipa a los sujetos tanto dentro como fuera del aula (Núñez, 2005).

En conclusión, la pedagogía crítica se presenta como proceso educativo que construye colectivamente el conocimiento y cambia las interacciones dentro del aula, además, transforma las relaciones del alumno con su contexto. Así, la educación es problematizadora (Freire, 1970); pues, en efecto, la creación dialógica del saber promueve la participación, la motivación y el surgimiento de aprendizajes significativos para el educando. Así, el proceso educativo adquiere una nueva dimensión ante sus ojos pues le permite reconocerse como ciudadano socialmente activo y constructivo. De hecho, garantiza compromisos reales con respecto al logro de la equidad, la inclusión y el desarrollo social. Es decir, la reflexión colectiva en el aula se transforma en acción crítica sobre la realidad.

\section{La educación con sentido humanista}

De acuerdo con la unESCO la educación debe ser un proceso desarrollado a lo largo de la vida que permita aprender a conocer, aprender a hacer, aprender a vivir y aprender a ser (1996). A través de esta propuesta, se espera que la educación garantice una formación integral y cuyo proceso incluya la adquisición de saberes, valores y habilidades. El objetivo es que le dé al individuo las herramientas para conocerse, desarrollar aprendizajes, ponerlos en práctica en el marco de situaciones sociales o laborales concretas, reflexionar críticamente sobre su contexto e incidir sobre sí mismo. Así, no solo basta con adquirir conocimientos sino que es necesario fomentar el desarrollo integral como ser humano, favorecer aspectos éticos y actitudes que promuevan la participación y el compromiso con las realidades sociales (Ruiz, Álvarez y Pérez, 2008). Debe ser entonces un proceso que tenga en cuenta la dimensión intelectual, humana y social del individuo.

En esa medida se hace referencia a un proceso formativo que incluye transversalmente distintas dimensiones de la vida humana y se relaciona con el ámbito de lo individual y lo colectivo. En primer lugar, se hace referencia a la posibilidad de promover la comprensión de sí mismo y de favorecer interacciones positivas con el entorno. En efecto, la educación con sentido humanista se centra en la persona y considera que su desarrollo 
debe ser integral, considerando aspectos intelectuales, afectivos y sociales, promoviendo la empatía, el trabajo en equipo y las relaciones interpersonales (Aizpuru, 2008). Por consiguiente, se fomenta la construcción de valores que permitan descubrir al otro, aprender desde la diferencia y fomentar mejores procesos de convivencia.

En segundo lugar, se percibe la posibilidad de promover una formación crítica y reflexiva para garantizar la conciencia y la responsabilidad del individuo frente a las necesidades de su comunidad. Además, se espera que esto garantice actuaciones concretas, individuales o colectivas, frente a esas circunstancias. En esa medida, la educación humanista debe garantizar un proceso de formación holístico e integral que favorezca aspectos sociales, emocionales, mentales y morales para que el sujeto consiga intervenir sobre las realidades de su contexto social (Wompner, 2008). Con ello se contribuye a fortalecer la autorrealización y la actuación colectiva frente a los intereses comunes. Por tanto, esta debe fomentar el autoconocimiento, la sensibilidad social, la interacción con su entorno y la acción comprometida frente a él para responder a sus problemáticas (Aizpuru, 2008; Ruiz, Álvarez y Pérez, 2008; Wompner, 2008). De este modo, el ser humano convierte el saber en experiencia vital y hace ejercicio de su papel como ciudadano.

\section{El rol del docente en los procesos educativos}

En esta instancia es pertinente destacar el papel de la institución educativa y del educador al momento de garantizar la calidad de la educación. De esta manera, surge la idea de la eficacia como práctica orientada a garantizar la continua mejora en los procesos de aprendizaje con el fin de optimizar el centro educativo y la labor de los docentes, y de impactar positivamente en los estudiantes (Barba, 2007; Murillo, Martínez y Hernández-Castilla, 2011). Así, la escuela debe generar una dinámica de retroalimentación de las prácticas docentes y del proyecto educativo institucional con el fin de garantizar un mejoramiento continuo. Para esto se puede aportar desde el trabajo en equipo dentro del establecimiento escolar y desde la construcción de un currículo que desarrolle competencias cognitivas, emocionales y prácticas (Braslavsky, 2006).

También es pertinente destacar el rol del educador en el ámbito educativo y en los procesos de formación. Más allá de entregar un conjunto de conocimientos que existen por sí solos, debe promover la construcción de esos saberes para incentivar el empoderamiento del estudiante. Su misión reside en hacer descubrir al alumno sus potencialidades con el fin de que pueda construir su propio proyecto de vida a partir de decisiones autónomas y responsables. Consiste, ante todo, en fomentar destrezas para alcanzar los objetivos y metas individuales, contribuyendo activamente al desarrollo 
sostenible de la sociedad. Desde este punto de vista, los profesores y los alumnos son ciudadanos que aprenden colectivamente y contribuyen juntos en los procesos de trasformación social (Ayuste et al., 1994).

Por ello, la labor docente adquiere una doble dimensión. En primer lugar, debe suscitar prácticas pedagógicas y didácticas que contribuyan a crear aprendizajes pertinentes, relevantes y transformadores para el educando. Reside en este objetivo la necesidad de generar experiencias productivas e innovadoras que contribuyan a mejorar el desempeño académico (Braslavsky, 2006). No obstante, el resultado de este tipo de educación no solo se refleja en términos de calidad, sino que también impacta en el alcance de los objetivos y logros de todo proceso educativo. Para ello, los docentes deben orientar la educación hacia la resolución de problemas prácticos, reales y cotidianos (Krichesky et al., 2011).

En segundo lugar, corresponde a los docentes fomentar no solo la consciencia acerca de sí mismo, sino también del propio contexto familiar y social. De esa forma, a partir de la comprensión crítica de la realidad, los estudiantes, en su calidad de ciudadanos, serán sujetos activos en la transformación de su propio contexto (Cochran-Smith, Gleeson y Mitchell, 2010; Conklin, 2008; Darling-Hammond, 2002). En estas circunstancias, el conocimiento no es una compilación de saberes sino una construcción. Los estudiantes no son receptores sino sujetos activos del proceso de enseñanza y aprendizaje. No hay pasividad sino participación orientada al debate, a la deconstrucción y a la creación de una nueva comprensión. A su vez, esta no es abstracta, sino que se manifiesta a partir de competencias reales para participar activamente en la sociedad. He ahí la transformación de la realidad a partir de la educación como proceso que, según Freire (1970), implica la conciencia crítica, la reflexión y la acción.

\section{Metodología}

\section{Tipo de investigación y técnicas para la recolección de los datos}

Esta investigación es de tipo cualitativo y como técnica para obtener la información se utilizó la entrevista. Esta sirve para estimular el flujo de datos y consiste en comprender los hechos y las percepciones desde el punto de vista del entrevistado para dimensionar los significados de sus experiencias vitales (Álvarez-Gayou, 2003; Woods, 1987). De esta manera, permite descentrarse por un momento del propio yo para acceder objetivamente al sistema de valores, creencias e ideas del informante. Así, debe contar con tres características: neutralidad con respecto al propio 
marco referencial, deseo de conocer las percepciones de las personas sobre sus hechos e historias, y la capacidad de percibir lo que dicen para evitar la distorsión (Woods, 1987). Por ello, es imprescindible reflejar esas posiciones y garantizar la veracidad al momento de mostrar los resultados del análisis.

En este caso particular, se utilizó la entrevista en profundidad como proceso que consiste en una conversación detallada entre entrevistador e informantes, dirigido hacia la comprensión de las perspectivas que ellos tienen sobre sus propias vidas, experiencias o situaciones (Taylor y Bogdan, 1987). Es decir, al contrario del formato tradicional de preguntas y respuestas, se desarrolla un ambiente distendido en el que ambas partes comparten sus experiencias a partir de un diálogo entre iguales. Más allá de recopilar datos, se trata de aprender mutuamente a partir de las historias vitales del otro.

\section{Problema de investigación y objeto de estudio}

El objeto de estudio de esta investigación se centra en las prácticas pedagógicas, didácticas y curriculares de docentes pertenecientes a una institución educativa del municipio de Carepa, región de Urabá, departamento de Antioquia (Colombia). Para ello, se pretende identificar las dificultades, estrategias y retos de los docentes entrevistados al momento de incidir en la construcción del proyecto de vida de sus estudiantes.

\section{Análisis y sistematización de los datos}

Para este análisis, se hizo una triangulación de los datos, técnica que permite la articulación de toda la información obtenida en la investigación para obtener una comprensión más clara del problema estudiado (Taylor y Bogdan, 1987). De esta manera, se tabularon los datos relacionados con las características de la población participante para luego caracterizarlos. Posteriormente, se leyó la información obtenida en las entrevistas a los docentes. A partir de ahí, se codificaron los datos; en particular, se reunieron y analizaron aquellos que se refieren a un tema, concepto e idea común (Taylor y Bogdan 1987; Álvarez-Gayou 2003; Fernández, 2006). Este procedimiento dio origen a una matriz de análisis compuesta por seis categorías: dificultades relacionadas con el contexto social; dificultades relacionadas con el sistema educativo; dificultades en el contexto rural; estrategias; resultados, y retos del docente y de la institución educativa. Esto se complementó con varias subcategorías por cada una, con el fin de identificar los hallazgos principales. Por último, para presentar los resultados, los datos se analizaron a la luz de la información cualitativa obtenida en las entrevistas a los docentes. 


\section{Caracterización de los docentes}

En cuanto a la caracterización de los docentes, se trata de seis maestros, el coordinador académico y el rector de la Institución Educativa El Playón, ubicada en el municipio de Carepa, región de Urabá, departamento de Antioquia, Colombia. De estos, se entrevistaron 5 hombres y 3 mujeres, correspondientes al $62 \%$ y al $38 \%$, respectivamente. De ellos, el $50 \%$ está en un rango de edades entre los 25 y 30 años, y el otro $50 \%$ tiene más de 30 años.

En cuanto a las profesiones, cuatro son licenciados. De acuerdo con el Ministerio de Educación Nacional (MEN, 2014), un licenciado es quien ha cursado una licenciatura en una institución de educación superior, y ha adquirido las habilidades para desempeñar la profesión docente. Se desarrollan licenciaturas en las cuatro áreas de la formación básica en el país: ciencias sociales, ciencias naturales, matemáticas y español. Sin embargo, también hay en otras, como inglés, educación física, etnoeducación, ética, religión, tecnología, pedagogía y primera infancia.

Cabe aclarar que, para adelantar este estudio, la entrevista se realizó con docentes que han ocupado distintos cargos durante su desempeño profesional, debido a su amplio conocimiento del sistema educativo en la región y con el fin de obtener una visión desde la perspectiva docente y directiva. Esto sirvió para enfatizar en la gestión académica y la gestión directiva de la educación básica colombiana. De acuerdo con este planteamiento, la gestión directiva hace referencia a la manera en la que la institución educativa es direccionada por partes de los directivos y su equipo de trabajo. La gestión académica hace referencia a los procesos pedagógicos y curriculares que esta desarrolla (MEN, 2008). Esto se complementa con la gestión administrativa que hace referencia a la manera de gestionar sus recursos económicos, físicos y humanos; y se complementa con la gestión de la comunidad, que alude a las estrategias desarrolladas para interactuar con su entorno social (MEN, 2008). Así, $37 \%$ de los entrevistados se ha desempeñado como docente y coordinador; $25 \%$ se ha desempeñado como docente, y otro $25 \%$ como coordinador, sumando el $50 \%$ de los entrevistados. Por su parte, solo uno de ellos se ha desempeñado como rector $(13 \%)$.

De igual manera, para obtener un panorama amplio sobre el papel de los docentes y del sistema educativo en el Urabá frente a los procesos académicos, se optó por docentes que se han desempeñado en los dos ciclos de la educación básica obligatoria en Colombia: primaria y bachillerato. De esta manera, $62 \%$ se ha desempeñado en bachillerato y primaria; $25 \%$ en primaria, y $13 \%$ en bachillerato. 


\section{Matriz de análisis}

Una vez se hizo la caracterización de los docentes participantes, se procedió con una matriz de análisis, conformada por las categorías y las subcategorías derivadas del proceso de estudio de los datos. Esto se representa en la tabla 1 .

\section{Tabla 1}

Matriz de análisis

Matriz de análisis

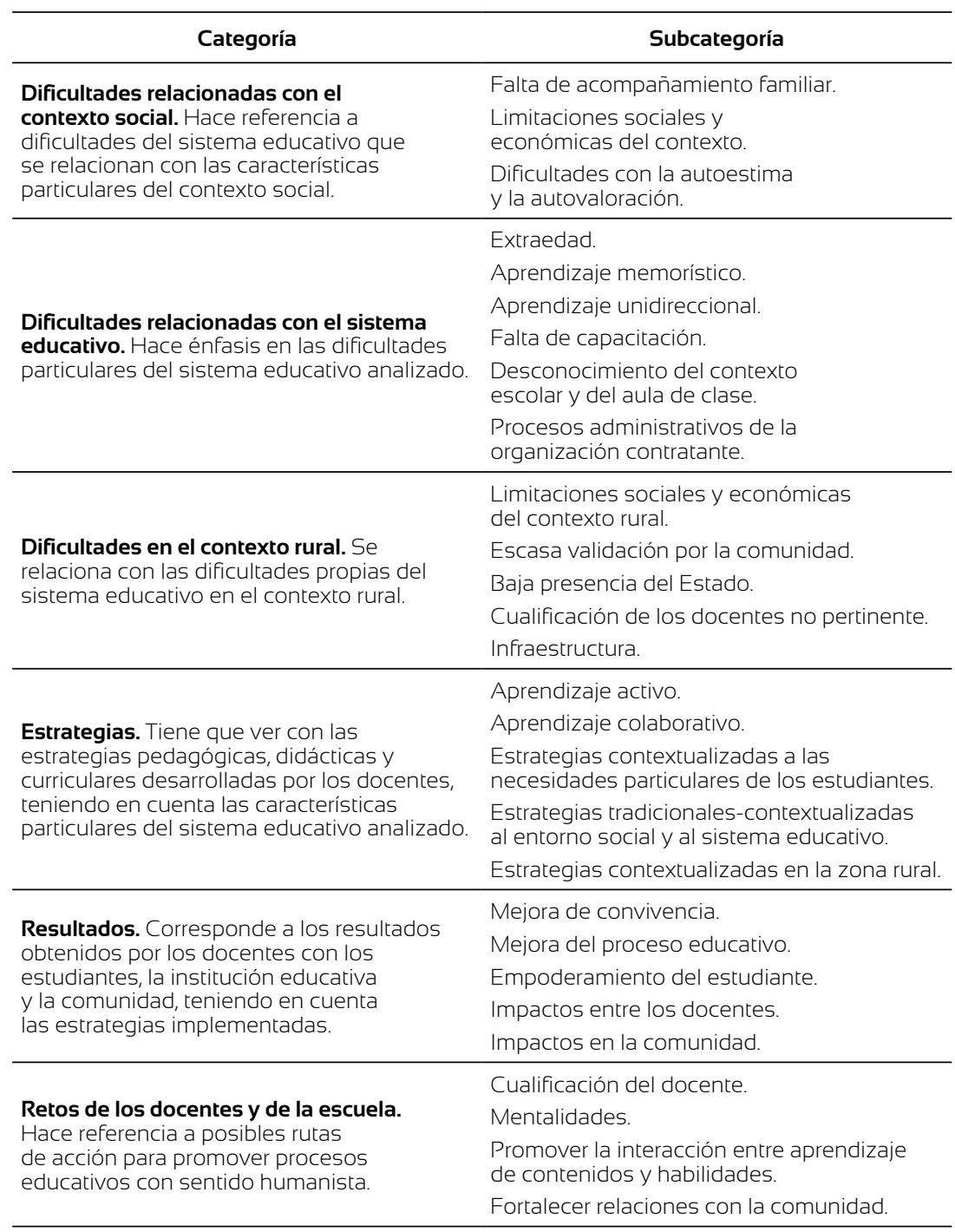

Fuente: elaboración propia. 


\section{Resultados}

\section{Dificultades de los procesos educativos en el Urabá antiqueño}

Al analizar el papel del docente y del sistema educativo en el fortalecimiento de los proyectos de vida alternativos (PVA), se puede identificar que en el Urabá antioqueño hay tres factores que adquieren un rol en este proceso: dificultades relacionadas con el contexto social, con el sistema educativo y con las características propias de la educación en el entorno rural. A través de su interacción, se pueden identificar varias limitaciones de tipo social y educativo que en ocasiones dificultan los procesos educativos desarrollados en la región. A continuación se analizan los tres elementos.

\section{Factores relacionados con el contexto social}

En cuanto a los factores relacionados con el contexto social, se pueden detectar tres dificultades: falta de acompañamiento familiar, baja autoestima y limitaciones sociales y económicas propias del contexto. Estos tres factores interactúan entre sí y delimitan las características de un contexto social vulnerable que incide directamente en las dificultades y limitaciones del sistema educativo analizado.

Con respecto al primer elemento, de acuerdo con los docentes entrevistados, es común identificar en algunos padres una falta de participación activa en los procesos educativos de sus hijos. Esto hace que algunos niños no tengan una figura adulta que garantice una supervisión de las actividades desarrolladas por sus hijos en la institución educativa. De esta manera, la ausencia de una figura en el hogar que represente la autoridad y que haga un seguimiento a su proceso educativo, repercute en problemas de convivencia escolar o dificultades de tipo académico. Todo ello confluye en un sentido de desmotivación frente al propio proceso educativo que se evidencia en la pérdida de asignaturas y grados escolares o en las dificultades para socializar con sus pares.

Como consecuencia de la ausencia o del poco acompañamiento familiar en la formación, el segundo elemento hace referencia a las dificultades relacionadas con la autoestima y la autovaloración. De igual manera, estas problemáticas encontraban también su explicación por los problemas familiares derivados de las propias estructuras sociales de la región. Así, esta ausencia familiar a veces estaba explicada también por el abandono de uno de los dos padres, la muerte de uno de ellos a veces en circunstancias violentas o por la necesidad de trabajar todo el día para 
conseguir el sustento del hogar. Por ello, algunos estudiantes respondían con la subvaloración de sus propias habilidades, con inseguridad frente a sus propios saberes, con retraimiento o con dificultades para convivir con sus compañeros.

Por último, el tercer elemento engloba los dos anteriores y tiene que ver con las limitaciones sociales y económicas del contexto. De esta manera, es pertinente recalcar que esa falta de acompañamiento familiar está asociada a las características propias del entorno social. Así, es común encontrar hogares desestructurados debido a las largas jornadas laborales, a la conformación de nuevas familias o a la muerte de alguno de sus integrantes en circunstancias violentas. Esto hace que la convivencia en el hogar también sea compleja y que estas problemáticas se reflejen en el centro educativo.

En consecuencia, las limitaciones sociales y económicas propias del contexto generan problemáticas que dificultan el acceso y el mantenimiento en el sistema educativo. Se trata de circunstancias como el pago de matrículas, la alimentación y el costeo de materiales escolares y transporte. Todo esto se dificulta debido al desempleo o, por el contrario, a las largas jornadas laborales de los padres de familia. Esto último también influye en la falta de acompañamiento familiar, que a veces genera en el estudiante ausentismo, repitencia, fracaso escolar, baja autoestima o agresividad. Por ello, al momento de analizar las dificultades relacionadas con el sistema educativo en el Urabá antioqueño, es imprescindible considerar el papel del contexto social.

\section{Factores relacionados con el sistema educativo}

En cuanto a factores relacionados con el sistema educativo, se encuentran seis dificultades: extraedad, aprendizaje memorístico, aprendizaje unidireccional, desconocimiento del contexto escolar, falta de capacitación y procesos administrativos de la organización contratante. A continuación, se analiza cada uno de ellos.

Con respecto a la extraedad, es común encontrar en la región esta circunstancia en la que los estudiantes no están cursando un grado académico en coherencia con su edad. Según el sistema educativo colombiano, el grado preescolar se cursa a los 5 años; la primaria, entre los 6 y los 10 años, y el bachillerato, entre los 11 y los 16 años. No obstante, en el caso de Urabá, esta problemática es frecuente debido a tres causas: a) son estudiantes que fueron desplazados desde sus lugares de origen como consecuencia del conflicto armado; b) sus familias han cambiado de lugar de residencia por razones laborales o reestructuración de los hogares, y c) han perdido el grado escolar en varias oportunidades como consecuencia de lo mencionado en el apartado de los factores sociales: falta de acompañamiento, baja autoestima 
y limitaciones económicas. Esa es una de las situaciones que explica los problemas de convivencia y la repitencia de años escolares en algunos casos. Pues en efecto, los estudiantes mayores no se sienten en confianza para socializar con sus compañeros y son excluidos, no tienen las habilidades para cursar el grado académico en el que están o sus inquietudes superan las exigencias de ese grado escolar.

Al lado de esta problemática, vienen dos elementos ligados a las características del sistema educativo tradicional en el Urabá antioqueño: el aprendizaje memorístico y el aprendizaje unidireccional. Ambos inciden para que el estudiante no sea un sujeto activo de su aprendizaje, sino un receptor que acumula conocimientos, tal y como lo sintetiza Paulo Freire con su concepto de educación bancaria. En el contexto social analizado, esta era la práctica educativa imperante hasta hace unos años. Sin embargo, actualmente en el departamento de Antioquia, se están realizado diversos esfuerzos por mejorar la calidad educativa frente a estos condicionamientos sociales e históricos. El caso analizado en esta investigación es uno de ellos.

De esta manera, el aprendizaje memorístico se desarrolla a través de una práctica tradicional donde el objetivo del estudiante consiste en ser capaz de memorizar, repetir, escribir y verbalizar conceptos como evidencia de su aprovechamiento. Esto entra en contradicción con la idea de la educación como un proceso que permita desarrollar habilidades y capacidades para la vida. Así, de alguna manera, se explica la problemática de la repitencia y la pérdida reiterada de asignaturas pues denota la necesidad de poner en práctica alternativas que permitan al estudiante utilizar saberes en contextos reales y cotidianos. De este modo, se fortalecería la motivación frente al propio proceso educativo.

De la mano del aprendizaje memorístico, va ligado el aprendizaje unidireccional. Aquí el docente se convierte en el único referente de autoridad, delimitando las características de un sistema educativo vertical, que parte de arriba hacia abajo y evita la posibilidad de construir saberes colectivamente. En estas circunstancias, el maestro representa la idea de una verdad absoluta y ya construida, anulando la posibilidad de la crítica y el disenso. Optar por una perspectiva contraria solo sería prueba del error del estudiante y no una oportunidad de construir conocimientos a partir del desarrollo de habilidades para reflexionar y analizar situaciones en contexto.

Posteriormente, surgen dos dificultades relacionadas entre sí y que se vinculan con las características de los docentes en el entorno educativo analizado. Se trata de la falta de capacitación y del desconocimiento del contexto escolar. La interacción de ambas circunstancias hace que, en ocasiones, ellos no tengan las herramientas para desarrollar procesos educativos con calidad. 
De este modo, con respecto a la falta de capacitación, es posible evidenciar que los docentes no tienen la posibilidad de acceder a procesos de formación y capacitación continua. Esto entra en contradicción con las dinámicas de las grandes ciudades del departamento, donde ellos tienen la oportunidad de recibir distintos cursos y talleres formativos. Esto hace que en algunas instituciones educativas no se desarrollen procesos de seguimiento, evaluación, y mejoramiento de las prácticas educativas realizadas.

En línea con la anterior dificultad, el desconocimiento del contexto escolar y del aula de clase se convierte en otro elemento que genera algunas limitaciones en los procesos educativos. En esta instancia influyen dos circunstancias que se relacionan entre sí. En primer lugar, la falta de un diagnóstico sobre las características sociofamiliares y académicas de los estudiantes para conocer las problemáticas que poseen y pueden interferir en su formación. De acuerdo con el análisis de los obstáculos relacionados con las limitaciones sociales y económicas del contexto, estos elementos pueden interferir en el éxito académico del estudiante. Esto se evidencia de una manera mucho más marcada en un contexto vulnerable como el observado. En segundo lugar, ese desconocimiento del entorno social y familiar del educando a veces tiende a promover las enseñanzas de contenidos que requieren mayor pertinencia con respecto a los conocimientos y las realidades personales de los estudiantes. Esa es tal vez un modo de generar mayores niveles de motivación y aprendizajes significativos.

Por último, existe una dificultad relacionada con los procesos administrativos desarrollados por la entidad contratante. En el Urabá antioqueño, algunas instituciones educativas funcionan bajo cobertura. Es decir, una modalidad de educación por contrato, desarrollada principalmente en contextos vulnerables o alejados de las principales zonas urbanas, donde el Estado contrata a instituciones privadas con el fin de prestar el servicio educativo (MEN, 2009). Esto representa una dificultad por dos razones. Primero, el proceso de contratación entre la Gobernación de Antioquia y las entidades privadas a veces se desarrolla entre diciembre y primeras semanas del año siguiente. Esto hace que el inicio del curso escolar se retarde casi un mes e inicie a finales febrero, al contrario que la mayoría de las instituciones educativas públicas colombianas, cuyas actividades académicas comienzan las últimas semanas de enero. De hecho, esta situación se dio en los años 2016 y 2017. Segundo, de acuerdo con algunos de los docentes, en ocasiones eso constituye un obstáculo, puesto que a veces estas entidades no poseen unos procedimientos administrativos claros y coherentes con sus objetivos misionales.

Con ello se evidencia que el sistema educativo tradicional en el Urabá antioqueño requiere cuatro necesidades que surgen de los factores mencionados anteriormente: a) optimizar los procesos de capacitación y 
formación continua de docentes para que mejoren sus prácticas pedagógicas, didácticas y curriculares; b) desarrollar estrategias educativas que transformen las tradicionales para responder a los procesos de aprendizaje unidireccional y memorístico que favorecen la acumulación de conocimientos sobre el desarrollo de habilidades; c) desarrollar procedimientos administrativos que sean claros, coherentes y pertinentes a las realidades de los estudiantes, y d) implementar estrategias concretas frente a la problemática de la extraedad para responder de manera asertiva ante los estudiantes que se encuentran en esta situación. Todo esto como manera de comenzar a analizar críticamente el sistema educativo de la región e identificar oportunidades de mejora.

\section{Factores relacionados con características del sistema educativo en el contexto rural}

Aquí se pueden encontrar cuatro dificultades: particularidades propias de las estructuras sociales, infraestructura, baja presencia del Estado y cualificación no pertinente de los docentes. La interacción de estas circunstancias hace que estas dificultades sean más complejas en contraste con las realidades educativas de la zona urbana.

La primera de estas dificultades tiene que ver con las limitaciones sociales y económicas propias de un contexto rural. En estas circunstancias, debido al difícil acceso a las instituciones educativas, a los escasos medios de transporte o a la precaria infraestructura en estos entornos, la presencia de los padres de familia es reducida. De esta manera, se encuentra una coincidencia con la falta de acompañamiento familiar en el ámbito urbano.

Precisamente, como consecuencia de la baja presencia familiar en las instituciones educativas, el segundo obstáculo se relaciona con la escasa validación por parte de la comunidad sobre los procesos académicos. En efecto, la educación no es un tema principal en cuanto a las discusiones sobre su posible impacto en el desarrollo integral de los jóvenes. Esto hace que la comunidad no se involucre activamente en los procesos de seguimiento y acompañamiento a las actividades desarrolladas en los centros escolares.

En coherencia con la segunda dificultad, la tercera de ellas se relaciona con la baja presencia del Estado en los procesos educativos realizados en la zona rural del Urabá antioqueño. En efecto, si no hay acciones ciudadanas concretas de movilización y organización en favor de la educación, esta tampoco tiene una presencia primordial en las políticas públicas y educativas desarrolladas en la zona. De esta manera, si bien en los últimos años desde la Gobernación del Departamento de 
Antioquia se han implementado estrategias para favorecer a la población en temas educativos, aún es necesario aumentar la inversión de recursos en infraestructura y cualificación de docentes.

De hecho, los dos últimos obstáculos se relacionan con esta problemática. Por tanto, el penúltimo de ellos tiene que ver con la cualificación no pertinente de los docentes que imparten procesos de enseñanza en el área rural. Particularmente, esto sucede porque el enfoque educativo más usado en las escuelas de educación primaria de estas zonas es el modelo escuela nueva, el cual consiste en la enseñanza en un solo salón de clases, de los diferentes grados y asignaturas de ese ciclo escolar. Así, esa dificultad surge por dos circunstancias: a) algunos docentes que llegan a estas escuelas provienen de contextos urbanos $y$, aunque han aprendido los contenidos en las universidades, necesitan las herramientas concretas para implementarlo en la práctica; b) se requiere aumentar la inversión por parte del departamento y de las escuelas en procesos de formación y capacitación de los docentes para que puedan enseñar los contenidos con pertinencia al contexto social.

Para terminar, la escasa inversión pública en procesos educativos en el ámbito rural es la causa de la última de las problemáticas que enfrentan los centros escolares en estas zonas: el deterioro de la infraestructura. Es común encontrar espacios donde no hay acceso al agua, los baños no funcionan y los materiales de estudio como sillas, pizarras y libros no existen, hay pocos o están en mal estado.

Así, en la zona rural se evidencian unas condiciones más complejas en comparación con el área urbana precisamente por las limitaciones sociales y económicas de ese contexto. Por ello, también es pertinente mejorar el acompañamiento familiar y fomentar la validación de los procesos educativos por parte de la comunidad. También, se requiere que el Estado aumente su presencia a través de la inversión pública para cumplir con dos objetivos: a) promover procesos de cualificación y formación continua de docentes y b) garantizar la mejora de la infraestructura y la dotación de materiales escolares para fomentar ambientes de aprendizajes saludables. Todo esto para generar prácticas educativas pertinentes a las características del ámbito rural.

\section{Estrategias y resultados de las prácticas desarrolladas frente a las limitaciones del sistema social y educativo}

De lo anterior se desprenden dos estrategias para favorecer los procesos educativos de los estudiantes en contextos vulnerables. Se trata de la combinación del aprendizaje activo con el aprendizaje colaborativo; en el primero, el estudiante se asume como protagonista de su formación; el segundo, promueve una construcción colectiva del conocimiento con

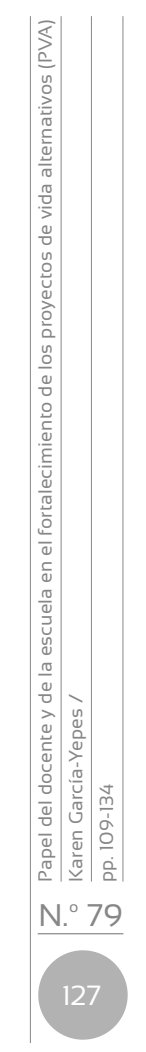


el fin de convertir el aula en un espacio de participación y ejercicio del liderazgo. La interacción de ambos sería una alternativa innovadora ante las prácticas educativas tradicionales del Urabá antioqueño.

Ante estas dos estrategias, surge la necesidad de promover aprendizajes contextualizados para promover procesos educativos pertinentes a las realidades sociales y a los conocimientos previos de los alumnos. Esto puede realizarse teniendo en cuenta tres aspectos, partiendo de lo general a lo específico: a) las características del entorno social y el sistema educativo del Urabá antioqueño; b) las carencias y limitaciones propias del contexto rural, y c) las necesidades particulares de los estudiantes.

Por ello, es evidente la necesidad de fomentar procesos de aprendizaje activo y colaborativo como respuesta a un sistema educativo tradicional donde se valora la acumulación de conocimientos sobre el desarrollo de habilidades para la vida. Para esto, es pertinente tener en cuenta tres alternativas: a) contextualización de las estrategias a las necesidades particulares de estudiantes con dificultades de aprendizaje, ausencia de acompañamiento familiar o problemas de convivencia: b) desarrollo de prácticas educativas innovadoras pero contextualizadas a las características del entorno social y educativo; c) implementación de propuestas formativas pertinentes a las características sociales y económicas del contexto rural. Así, se percibe la posibilidad de responder de manera pertinente a las realidades de una comunidad en situación de vulnerabilidad.

Desde esta perspectiva, ante las estrategias desarrolladas por los docentes teniendo en cuenta estos obstáculos, se presentan unos resultados con impactos en tres actores, partiendo de lo específico a lo general: estudiantes, docentes y comunidad. A partir de la interacción entre los tres, se generan unos efectos significativos tanto en los alumnos como en la comunidad.

En esa medida, frente a los impactos relacionados con el estudiante, se produce un proceso de doble vía. Primero, se presenta un aporte significativo a los procesos de convivencia en el contexto escolar a partir de la puesta en práctica de estrategias concretas para resolver conflictos. Segundo, se promueve la construcción de aprendizajes significativos y el desarrollo de habilidades para aplicarlas en contexto. Todo ello confluye en el empoderamiento como alternativa para actuar de manera autónoma en favor de la construcción del proyecto de vida.

En cuanto a los impactos relacionados con los docentes, es posible percibir un proceso a través del cual se generan dinámicas de reflexión acerca de la responsabilidad que implica el rol del maestro debido a su papel central en la formación de los estudiantes. De igual modo, posibilita las condiciones concretas para sugerir propuestas pedagógicas como respuesta a los enfoques metodológicos asentados en la visión tradicional 
e instrumental de la educación. Esto es importante por cuanto crea un ambiente propicio para compartir experiencias y promover prácticas innovadoras en las estrategias de enseñanza/aprendizaje.

Por último, sobre los impactos relacionados con la comunidad, se evidencia un proceso a través del que padres de familia comienzan a asumir un papel más activo en las estrategias de enseñanza y aprendizaje. Esto resulta por las prácticas propositivas del docente y por la apropiación de la familia de su rol como primer escenario de socialización al ser vinculados por la institución educativa y por el maestro en los procesos educativos.

Desde esta perspectiva, para promover procesos educativos que generen impactos en los estudiantes, en los docentes y en la comunidad, es necesario suscitar prácticas didácticas, pedagógicas y curriculares contextualizadas; es decir, que sean coherentes con las necesidades particulares del sistema educativo para responder a los requerimientos de los estudiantes. Esto se presenta como estrategia para adelantar procesos formativos que den preferencia al desarrollo de habilidades sobre la acumulación de conocimientos. Así, se logran las condiciones para aplicar las capacidades aprendidas en contextos concretos y no solo en la resolución de exámenes académicos.

\section{Retos de los docentes frente a los procesos educativos en el Urabá antioqueño}

Del análisis de las dificultades que presenta el sistema educativo en el Urabá, así como de las estrategias y resultados derivados de las prácticas pedagógicas de los docentes que participaron en esta investigación, es posible plantear que en la región hay tres retos, los cuales involucran tres componentes, partiendo de los desafíos del docente y de la institución frente a la labor del maestro, al estudiante y a la comunidad. A partir de esa interacción sería viable iniciar procesos educativos pertinentes a las realidades y las problemáticas de un entorno social.

En cuanto a los retos relacionados con la labor del docente y a partir de las dificultades analizadas con respecto a los pocos procesos de capacitación continua, es pertinente desarrollar procesos que permitan una cualificación constante del maestro. Esto se presenta como alternativa para mejorar las prácticas pedagógicas, didácticas y curriculares en el aula. De la misma manera, se presenta como estrategia para responder a las prácticas tradicionales del sistema educativo en la región.

Frente a los retos del docente con respecto al estudiante, hay dos desafíos. Por un lado, el impacto en las mentalidades del estudiante, y por otro, el desarrollo de procesos que favorezcan el desarrollo de aprendizajes, capacidades y habilidades sociales. De esta manera, se garantizan procesos educativos pertinentes a las realidades sociales de los contextos. 
En esa medida, con respecto a las mentalidades, es necesario facilitarle al estudiante la reflexión sobre sí mismo, así como sobre su responsabilidad en la construcción de su propio proyecto de vida. Por ello, la evidencia de las problemáticas sociales del contexto analizado, así como de las prácticas tradicionales del sistema educativo de la región, no debe ser el obstáculo sino la causa que explica esta necesidad. En efecto, es pertinente promover estrategias concretas que permitan al estudiante reflexionar sobre su papel en la toma autónoma de decisiones y en la realización de acciones específicas en favor de sus objetivos vitales.

El otro desafío relacionado con el estudiante consiste en promover la interacción entre aprendizaje de contenidos y habilidades sociales. Es decir, en su formación el alumno no solo requiere recibir conocimiento, sino que también es conveniente adquirir habilidades para aplicarlas en contextos sociales y cotidianos. Esto se convierte entonces en una alternativa para promover prácticas educativas coherentes con las necesidades de los estudiantes y las características del contexto social.

Por último, el reto de los docentes y de las instituciones educativas con respecto a su entorno social consiste en fortalecer las relaciones entre la escuela y la comunidad. Esto es necesario por cuanto el centro escolar también es un componente de las organizaciones que integran un contexto. Desde esta perspectiva, sus dinámicas también son causa de las problemáticas que se viven en las comunidades, y las que se viven en el plantel también reflejan esas realidades sociales. Por ello, corresponde potenciar la relación entre la institución educativa y la población con el fin de que esta incentive procesos pedagógicos y educativos que respondan a estas circunstancias. De esta forma, se podrá vincular activamente a alumnos, docentes, directivos y padres de familia en procesos de desarrollo comunitario desde la base.

Es pertinente plantear entonces que el sistema educativo en el Urabá antioqueño tiene tres retos con respecto al docente, al estudiante y a la comunidad: a) mejorar los procesos de cualificación y formación continua de los maestros; b) promover prácticas formativas del estudiante que hagan énfasis en la interacción entre conocimientos y habilidades, así como en la transformación de mentalidades, y c) fortalecer las relaciones con la comunidad para aumentar el acompañamiento familiar y la validación por parte de la población frente a las actividades desarrolladas por la escuela. Estas se constituyen en estrategias para fortalecer el papel del docente y del sistema educativo frente al contexto al que pertenecen. 


\section{Conclusiones: papel del docente y de la escuela en el fortalecimiento de los proyectos de vida alternativos (PVA)}

Al analizar el papel del docente y de la escuela en el fortalecimiento de los proyectos de vida alternativos (PVA), es pertinente mencionar que les corresponde incidir en el desarrollo de habilidades para la vida y en la aplicación de conocimientos en contexto. Esto para promover el acceso a todas las etapas de la educación básica obligatoria en condiciones de equidad con respecto a estudiantes de sistemas educativos privados. Igualmente, esto permitirá al educando buscar el desarrollo de trayectorias vitales que sean posibles en medio de los condicionamientos sociales y económicos propios de contextos vulnerables.

Es pertinente trascender a los discursos que se centran en la función reproductora de la educación a partir de una visión instrumental de la enseñanza. Este tipo de discursos, a pesar de su intención crítica, mantienen la visión sobre las características excluyentes de los procesos educativos en contextos vulnerables. Acá se obvia que la evidencia de la ausencia en cuanto a condiciones de calidad, es precisamente el reto frente al cual hay que debatir y plantear soluciones.

En efecto, no sería necesario hablar de la función social de la escuela, si el tema objeto de debate no lleva también a los actores involucrados, ya sean docentes, padres de familia, comunidad o agentes del Estado, a redefinir su papel frente al proceso formativo del estudiante. Es claro que la escuela debe responder de manera competitiva frente a estudiantes que han recibido contenidos curriculares y prácticas pedagógicas donde se valida la acumulación de conocimientos, sobre la construcción de saberes críticos y pertinentes a sus realidades sociales. Por ello, el desafío consiste en plantear estrategias concretas ante estas circunstancias para cambiar el papel del sistema educativo con respecto al estudiante e impactar en su proyecto de vida.

En conclusión, el papel del docente y de la escuela en el fortalecimiento de los PVA es ofrecer prácticas pedagógicas pertinentes al contexto social y educativo del estudiante. Ello debe ir orientado hacia el impacto en sus mentalidades y a la interacción entre el aprendizaje de conocimientos, valores y habilidades para la vida. En esa medida, se debe promover un proceso educativo con sentido humanista que fortalezca su dimensión intelectual, humana y social. Así, se logran las condiciones para que asuma un papel crítico y activo ante las realidades de su entorno e incida sobre él. Esto se constituye como alternativa para impactar en la construcción de los PVA. 


\section{Referencias}

Aizpuru, M. (2008). La persona como eje fundamental del paradigma humanista. Acta Universitaria, 18(1), 33-40.

Álvarez-Gayou, J. (2003). Cómo hacer investigación cualitativa. Fundamentos y metodología. México D.F.: Paidós.

Ayuste, A., Flecha, R., Palma, F. y Lleras, J. (1994). Planteamientos de la pedagogía crítica: Comunicar y transformar. Barcelona: Graó.

Ayuste, A. (2011). La educación transformadora en la pedagogía contemporánea. Crítica, 61(972), 16-20.

Ayuste, A. y Trilla, J. (2005). Pedagogías de la modernidad y discursos posmodernos sobre la educación. Revista de Educación, (336), 219-248.

Barba, B. (2007). Entre las revoluciones y la burocracia: Gobernar para la calidad de la educación. REICE: Revista Electrónica Iberoamericana sobre Calidad, Eficacia y Cambio en Educación, 5(3), 22-25.

Boyce, M. (1996). Teaching critically as an act of praxis and resistance. Electronic Journal of Radical Organization Theory, 2(2), 1-14.

Braslavsky, C. (2006). Diez factores para una educación de calidad para todos en el siglo xxI. REICE: Revista Electrónica Iberoamericana sobre Calidad, Eficacia y Cambio en Educación, 4(2), 84-101.

Casamitjana, M., Puigvert, L., Soler, M. y Tortajada, I. (2000). Investigar y transformar. Cultura y Educación, 17-18, 117-128.

Cochran-Smith, M., Gleeson, A. y Mitchell, K. (2010). Teacher education for social justice: What's pupil learning got to do with it? Berkeley Review of Education, 1(1), 35-61.

Conklin, H. (2008). Modeling compassion in critical, justice-oriented teacher education. Harvard Educational Review, 78(4), 652-674.

Darling-Hammond, L. (2002). Educating a profession for equitable practice. Learning to teach for social justice (pp. 201-217). Nueva York: Teachers College Press.

Fernández, L. (2006). ¿Cómo analizar datos cualitativos? Recuperado de http://www.ub.edu/ice/recerca/fitxes/fitxa7-cast.htm

Freire, P. (1970). Pedagogía del oprimido. Montevideo: Tierra Nueva.

Freire, P. (1976). Educación y cambio. Buenos Aires: Ediciones Búsqueda.

Freire, P. (1993). Pedagogía de la esperanza: Un reencuentro con la pedagogía del oprimido. Ciudad de México: Siglo xxı.

Freire, P. (1997). Pedagogía de la autonomía: Saberes necesarios para la práctica educativa. Ciudad de México: Siglo xxı.

Giroux, H. (2014). La pedagogía crítica en tiempos oscuros. Praxis Educativa, 17(2), 13-26. 
Goodman, G. (2008). Educational psychology: An application of critical constructivism. Nueva York: Peter Lang Publishing.

Grundy, S. (1987). Curriculum: Product or praxis. Nueva York: The Falmer Press.

Krichesky, G., Martínez-Garrido, C., Martínez, A., García, A., Castro, A. y González, A. (2011). Hacia un programa de formación docente para la justicia social. REICE: Revista Electrónica Iberoamericana sobre Calidad, Eficacia y Cambio en Educación, 9(4), 63-77.

McLaren, P. (2005). La vida en las escuelas: Una introducción a la pedagogía crítica en los fundamentos de la educación. México: Siglo xxı.

Ministerio de Educación Nacional (MEN) (2008). Guía para el mejoramiento institucional. De la autoevaluación al plan de mejoramiento. Bogotá.

Ministerio de Educación Nacional (MEN) (2009). Contratación del servicio educativo. Recuperado de http://www.mineducacion.gov.co/1621/ w3-article-248079.html

Ministerio de Educación Nacional (MEN) (2014). Lineamientos de calidad para las licenciaturas en educación. Recuperado de http://www.mineducacion.gov.co/1759/articles-357233_recurso_1.pdf

Murillo, F., Martínez, C. y Hernández-Castilla, R. (2011). Decálogo para una enseñanza eficaz. REICE: Revista Electrónica Iberoamericana sobre Calidad, Eficacia y Cambio en Educación, 9(1), 6-27.

Núñez, C. (2005). Educación popular: Una mirada de conjunto. Decisio, 10, 3-14.

Ruiz, J., Álvarez, N. y Pérez, E. (2008). La orientación sociohumanística, un aporte a la formación integral del estudiante. Tendencias Pedagógicas, 13, 175-191.

Taylor, S. y Bogdan, R. (1987). Introducción a los métodos cualitativos de investigación. Barcelona: Paidós.

UNESCO (1996). La educación encierra un tesoro. Informe para la UNESCO de la comisión internacional sobre la educación para el siglo XXI. Madrid: Santillana - UNESCO.

Wompner, F. (2008). Inteligencia holística. la llave para una nueva era. Barcelona: Parnass Ediciones.

Woods, P. (1987). La escuela por dentro: La etnografía en la investigación cualitativa. Barcelona: Paidós. 